\section{Von der anspruchsvollen Kunst der Abhängigkeit}

\section{Hein₹ Rüegger}

Für die meisten Menschen des westlichen Kulturkreises ist Freiheit im Sinne von Unabhängigkeit ein hohes Gut. ${ }^{1}$ Wir möchten möglichst autonom, selbstbestimmt und selbstständig leben. Von anderen abhängig zu sein, gilt in westlichen Gesellschaften nach einer langen moralphilosophischen Tradition weithin als ein Zeichen von Schwäche und Inkompetenz, als etwas Beschämendes und Entwürdigendes, das es wenn irgendwie möglich zu vermeiden gilt. ${ }^{2}$ Denn westlich-liberale Gesellschaften sind ganz auf Unabhängigkeit fokussiert, auf Autonomie und Kontrolle über alles, was geschieht, sosehr, dass diese Haltung nach dem Urteil des Medizinethikers Daniel Callahan schon «fast die Qualität einer Besessenheit angenommen hat.» ${ }^{3}$ Autonomie verstanden als Selbstständigkeit in der Bewältigung des Alltags wird verabsolutiert und scheint vielfach geradezu als Voraussetzung für eine menschenwürdige Existenz zu gelten. Die Angst davor, von anderen abhängig zu werden, auf fremde Hilfe angewiesen zu sein und andern zur Last zu fallen ist weit verbreitet. ${ }^{4}$ Sie zeigt sich insbesondere in der Angst vor zunehmender Abhängigkeit aufgrund der Begleiterscheinungen fortschreitenden Alters.

1 Dieser Aufsatz ist eine überarbeitete und erweiterte Version von Ausführungen, die zuerst unter dem Titel «Ist abhängig sein entwürdigend?» im Vögele Kultur Bulletin Nr. 108, 2019, 33-35 erschienen.

2 Alasdair C. MacIntyre, Dependent rational animals. Why human beings need the virtues, Chicago 1999, 2-7.

3 Daniel Callahan, Nachdenken über den Tod. Die moderne Medizin und unser Wunsch, friedlich zu sterben, München 1998, 18.

4 Thomas Klie weist darauf hin, dass andern zur Last zu fallen, den eigenen Angehörigen oder der Gesellschaft insgesamt, die grösste Befürchtung älterer Menschen is

Jahrbuch Diakonie Schweiz 4 (2020/2021) - ISSN 2504-3994

Dieser Text ist lizenziert unter einer Creative Commons Namensnennung 4.0 International Lizenz (CC BY 4.0): (https://creativecommons.org/licenses/by/4.0/).
Entsprechend schwierig ist es in einem solchen gesellschaftlichen Kontext, sich sein Angewiesensein auf die Hilfe anderer einzugestehen und von anderen Hilfe anzunehmen. Hinter dieser Schwierigkeit steht ein einseitiges, liberales Konzept von Autonomie, das Autonomie nur als Selbstständigkeit im Sinn von negativer Freiheit versteht: frei von jeder Einflussnahme durch andere und frei von jeglicher Abhängigkeit von anderen. Diese Vorstellung von Selbstständigkeit und Unabhängigkeit ist jedoch eine Abstraktion und wird der Realität menschlichen Lebens in keiner Weise gerecht. Sie ist - mit den Worten des amerikanischen Altersethikers Harry R. Moody - eher so etwas wie eine narzisstische Illusion. ${ }^{5}$

\section{Gegenseitige Abhängigkeit als Grundzug menschlichen Le-} bens

Aufgrund des leiblichen und sozialen Charakters menschlicher Existenz gibt es Selbstbestimmung und Selbstständigkeit nur zusammen mit Abhängigkeit. Ohne gegenseitige Verwiesenheit ist menschliches Leben nicht

Thomas Klie, Wen kümmern die Alten? Auf dem Weg in eine sorgende Gesellschaft, München 2014, 134). Vgl. dazu die Untersuchung von Sabine Pleschberger, Nur nicht zur Last fallen. Sterben in Würde aus der Sicht alter Menschen in Pflegeheimen, Freiburg 2005.

5 Harry R. Moody schreibt in The cost of autonomy, the price of paternalism, in: Robert Dish / Rose Dobof / Hary R. Moody (Hg), Dignity and A superficial reading of dependency risks making us oblivious to our common human fate: we were all once dependent, we will be so again, and we are so in manifold ways even at this moment. The blindness of adulthood is an intoxication with the illusion of independence. Perpetuating narcissistic illusions of independence - including noninterference - carries tremendous moral risks for a culture that idolizes independence and autonomy in every sphere of life, as ours does» (121). 
denkbar. ${ }^{6}$ Der amerikanische Moralphilosoph George J. Agich hat darum Recht, wenn er von einer fundamentalen anthropologischen Dialektik von Abhängigkeit und Unabhängigkeit spricht und darauf hinweist, dass wir uns immer schon in einem sozialen Gefüge vorfinden, das von Interdependenz, also von gegenseitigem Aufeinander-angewiesen-Sein bestimmt ist. ${ }^{7}$ Insofern ist Autonomie nicht als Gegensatz von Abhängigkeit zu verstehen, sondern als selbstverantwortliche Souveränität im Umgang mit Freiheiten und Abhängigkeiten, die beide gleichermassen zu jedem Leben gehören. ${ }^{8}$ Das mag in höherem Alter bei zunehmender Gebrechlichkeit besonders deutlich erfahrbar werden, gilt aber grundsätzlich auch im Blick auf die früheren Lebensphasen. Denn mit dem Ethiker Jean-Pierre Wils gilt es zu bedenken: «Unser Körper-Sein ist mit zahlreihen Abhängigkeiten behaftet - Abhängigkeiten, die uns zuallererst mit dem anderen in Verbindung setzen und uns mit einem gehörigen Mass an Passivität konfrontieren. Wir (empfangen das meiste, woraus wir leben und handeln. Es gibt ein Mass an Passivität, das unserer Handlungsautonomie einschränkend

6 Zum Folgenden vgl. Heinz Rüegger, Bevond Control. Dependence and Passivity in Old Age, in: Mark Schweda / Michael Coors / Claudia Bozzaro (Hg.), Aging and Human Nature. Perspectives from Philosophical, Theological, and Historical Anthropology (International Perspectives on Aging, Vol. 25), Berlin 2020, 47-57.

7 George J. Agich, Dependence and autonomy in old age. An ethical framework for long-term care, Cambridge 2003, 96.

8 Nach Gernot Böhme, Ich-Selbst. Über die Formation des Subjekts, München 2012 hat man in Europa alles daran gesetzt, Abhängigkeiten sowohl gegenüber der Natur als auch gegenüber anderen Menschen zu überwinden. In diesem Bestreben nach Emanzipation wurde geradezu die Entwicklung des Menschen zum Menschen gesehen. Dem stellt Böhme das japanische Konzept des Amae gegenüber, eine Form der Liebe, zu deren Kerngehalt die Zustimmung zu Abhängigkeit gehört. Und er macht deutlich: «Souverän ist man gerade dann, wenn man sich auch etwas widerfahren lassen kann, (...) wenn man sich in seiner Abhängigkeit akzeptieren kann» (242). zuvorkommt. Was zuerst kommt, ist nicht unsere Autonomie, sondern die geteilte Erfahrung der Abhängigkeit.»?

Was wir uns im Alltag meist nicht bewusst machen: Auf vielerlei Weise angewiesen zu sein, auf die natürlichen Lebensgrundlagen, auf Kultur oder auf die Zuwendung und Unterstützung von Mitmenschen, ist eine grundlegende Charakteristik menschlicher Existenz, ist essenzieller Teil der condition bumaine und als solcher gerade kein Makel. Im Gegenteil: Die Psychotherapeutin Ingrid Riedel hat schon 1991 in einem Aufsatz darauf hingewiesen, dass Abhängigkeit Ausdruck unseres gegenseitigen Aufeinander-bezogen-Seins als Mitmenschen ist und deshalb nichts Pathologisches, sondern etwas zutiefst Humanes, das es zu würdigen und zu kultivieren gilt. ${ }^{10}$ Sie spricht - bewusst provokativ! - von einer «Kunst der Abhängigkeit», die zu missachten einer Flucht vor wahrer Mitmenschlichkeit gleichkäme, weil für das Bestreben, Abhängigkeit grundsätzlich zu vermeiden, der Preis des Unbezogenseins, der Beziehungslosigkeit zu bezahlen wäre und unser Leben dadurch Schaden nähme. In diesem Sinne ist Daniel Callahan zuzustimmen: «Das Ziel, stets unabhängig zu sein, kann nur für begrenzte Zeit erreicht werden. Früher oder später, für längere oder kürzere Zeit, werden wir von anderen abhängig sein. Zumindest begleitet uns dieses Risiko immer, ist ein unentrinnbarer Teil unseres Lebens. Es ist ein ganz schwerwiegender Irrtum zu glauben, dass unser Wert als Person sinkt, weil Abhängigkeit unser Teil sein wird. Ein Selbst lebt in der ständigen Spannung zwischen Abhängigkeit und Unabhängigkeit. Beide sind ein Teil von uns. Die Unabhängigkeit mag uns ein besseres Gefühl geben.

9 Jean-Pierre Wils, Autonomie und Passivität. Tugenden einer zweiten Aufklärung im medizinischen Kontext, in: Ruth Baumann-Hölzle (Hg.), Leben um jeden Preis? Entscheidungsfindung in der Intensivmedizin, Bern 2004, 43-57 (45).

10 Ingrid Riedel, Die Kunst der Abhängigkeit, in: Peter Buchheim / Manfred Cierpka / Theodor Seifert (Hg.), Psychotherapie im Wandel. Abhängigkeit (Lindauer Texte), Berlin 1991, 197-211.
Jahrbuch Diakonie Schweiz 4 (2020/2021) - ISSN 2504-3994 CC by 4.0
Jahrbuch Diakonie Schweiz 4 (2020/2021) http://dx.doi.org/10.22018/JDS.2021.16 
Trotzdem bleibt sie nur die halbe Wahrheit unseres Lebens.» ${ }^{11}$ Die ganze Wahrheit unseres Lebens wird nur im bewusst angenommenen und gelebten Miteinander und Ineinander von Unabhängigkeit und Abhängigkeit erfahrbar. Wahrscheinlich erschliesst sich einem diese Einsicht besonders im höheren Alter in Auseinandersetzung mit abnehmenden Kräften und zunehmendem Unterstützungsbedarf. Hans-Martin Rieger weist darum mit gutem Grund in seinem Entwurf einer gerontologischen Ethik der Angewiesenheitsstruktur des Menschen, also seiner konstitutiven Abhängigkeit von anderen, eine zentrale Stellung zu und hält fest: «Im Alter wird die Angewiesenheitsstruktur konkret, die das ganze menschliche Dasein nicht lediglich beschränkt, sondern - positiv verstanden - auszeichnet.» ${ }^{12}$ Diese Angewiesenheit oder Abhängigkeit gilt es nach Rieger - ganz im Sinne von Riedels Postulat einer «Kunst der Abhängigkeit» - sowohl anzuerkennen als auch zu gestalten.

\section{Unterschiedliche Abhängigkeiten}

Natürlich gibt es unterschiedliche Formen von Abhängigkeit: bewusst eingegangene (etwa in arbeitsteiligen Kooperationsprozessen oder bei freiwilligem Bezug von Dienstleistungen professioneller Fachleute) und unfreiwillig aufgezwungene (etwa bei einem unumgänglich gewordenen Umzug in ein Pflegeheim), vorübergehende (etwa die Abhängigkeit kleiner Kinder von ihren Eltern) und permanente (etwa die Abhängigkeit von Menschen mit einer geistigen Behinderung gegenüber ihren Betreuenden). Während Erstere in der Regel als selbstverständlich und «normal» hingenommen und gar nicht unbedingt als Formen von Abhängigkeit wahrgenommen werden, gelten Letztere als notwendiger, aber problematischer

一

11 Callahan, Nachdenken über den Tod (Anm. 3), 176.

12 Hans-Martin Rieger, Altern anerkennen und gestalten. Ein Beitrag zu einer gerontologischen Ethik (Forum Theologische Literaturzeitung, Bd. 22), Leipzig 2008, 69.
Ausdruck einer persönlichen Schwäche oder Defizienz, an der man leidet und für die man sich möglicherweise sogar schämt. ${ }^{13}$ So ist es sicherlich richtig, unnötige, vermeidbare Formen von Abhängigkeit zu überwinden und Menschen zu einem möglichst hohen Mass an Selbstständigkeit und Selbstbestimmung (Autonomie) zu befähigen. Kinder sollen der Abhängigkeit von Eltern entwachsen und im Verlauf der Zeit lernen, ein eigenständiges Leben zu führen. Menschen mit Behinderung soll geholfen werden, in möglichst vielen Bereichen ihr Leben trotzdem selbstbestimmt zu gestalten.

Dennoch ist im Blick zu behalten, dass es menschlich normal ist, im Lebensvollzug auf vielfältige Weise der Hilfe und Unterstützung durch andere bedürftig zu sein. In vielen Situationen zeigt sich Autonomie nicht darin, dass Abhängigkeiten überwunden und Unabhängigkeit von anderen erreicht werden kann, sondern Selbstbestimmung erweist sich in der Art und Weise, wie jemand unüberwindbare Abhängigkeiten realistisch akzeptiert und so weit möglich selbst darüber befindet, wie er oder sie mit diesen Abhängigkeiten leben und welche Hilfe er oder sie in Anspruch nehmen will. Hier zeigt sich die von Ingrid Riedel postulierte «Kunst der Abhängigkeit»: im Unterscheidungsvermögen, welche Abhängigkeit zu überwinden und welche zu akzeptieren ist, und in der Haltung, wie jemand eigenverantwortlich mit Abhängigkeiten und dem sich daraus ergebenden Unterstützungsbedarf umgeht. Das Einüben einer solchen Kunst der Abhängigkeit ist anspruchsvoll, gehört aber ganz zentral zu jeder Lebenskunst, schon gar zu jeder Lebenskunst des Alters.

-

13 Es ist zurecht darauf hingewiesen worden, dass der sozioökonomische Status einer Person eine wichtige Rolle spielt bei der Art, wie Abhängigkeit gelebt und empfunden wird: «The privileged have their dependence needs met almost invisibly-meals appear, beds are made, bills paid, suits pressed - and in socially acceptable ways that honor norms of independence. They are snormalized and do not face threats to their adult status. The 84-year-old woman in a wheelchair is visibly dependent and is out of the mainstream, an anomaly» (Martha. B. Holstein / Jennifer A. Parks / Mark H. Waymack, Ethics, aging, and society. The critical turn, New York 2011, 115). 
Der Philosoph Gernot Böhme ersetzt das Ideal der Autonomie darum durch dasjenige der Souveränität. Für ihn ist der souveräne Mensch derjenige, der gerade nicht alles in seinem Lebensvollzug meint selber bestimmen, meistern und kontrollieren zu müssen, sondern der sich etwas widerfahren lassen kann, über das er nicht selber verfügt, und der gelassen mit seinen Abhängigkeiten umgehen kann. ${ }^{14}$ Ganz ähnlich versteht Wilhelm Schmid in seinen philosophischen Überlegungen zu einer Lebenskunst Selbstbestimmung als «ein aktiver ebenso wie ein passiver Prozess, ein Tun ebenso wie ein Hinnehmen und Lassen, ein eigenes Gestalten wie auch ein Sich-Gestaltenlassen von anderen, von Umständen und Situationen.» ${ }^{15}$ Die Bereitschaft zu solchem Sich-Gestaltenlassen von anderen ist ein zentraler Aspekt eines lebensfreundlichen, selbstbestimmten Umgangs mit Abhängigkeiten.

Angewiesen zu sein auf andere, ist so gesehen etwas zutiefst Humanes und Sinnhaftes, das jeder selbstbestimmten Lebensführung - schon gar, aber keineswegs nur im höheren Alter - als Ermöglichungsgrund vorausliegt. Es ist ein fundamentales Missverständnis zu glauben, Abhängigkeit von anderen sei ein existenzielles Unglück und sinnvolles, menschenwürdiges Leben nur möglich, wenn man sein Leben möglichst ohne Angewiesensein auf andere bewältigen kann.

\section{Leben im Modus des Empfangens}

Denkt und lebt man von einem solchen Verständnis von Angewiesenheit her, kann Abhängigkeit anders als blosse Kränkung unseres Narzissmus wahrgenommen werden, nämlich als Hinweis darauf, dass wir immer schon im Modus des Empfangens leben. Wir leben ganz wesentlich dank

14 Böhme, Ich-Selbst (Anm. 8), 58.242.

15 Wilhelm Schmid, Mit sich selbst befreundet sein. Von der Lebenskunst im Umgang mit sich selbst, Frankfurt a.M. 2004,119.
Jahrbuch Diakonie Schweiz 4 (2020/2021) - ISSN 2504-3994 dem und von dem, was uns von anderen zukommt, was sie an Hilfreichem zu unseren Lebensmöglichkeiten beitragen. Wir wären nicht, wer wir sind, und wir könnten nicht das Leben führen, das wir leben, wären da nicht andere, von deren Zuwendung, Rat und Unterstützung wir vielfältig profitieren. Insofern ist uns nicht nur unser Leben als solches grundsätzlich geschenkt - biologisch von den Eltern, theologisch gesehen von Gott -, sondern wir leben dauernd von der Hilfe anderer, von denen wir abhängig sind. Niemand lebt für sich allein und allein aus sich selbst. Wir leben immer als Beschenkte und Empfangende. Insofern hat der Theologe Fulbert Steffensky Recht, wenn er pointiert festhält: «Unabhängigkeit als Ideal ist die Selbstverdammung zur eigenen Dürftigkeit. Ich muss mein eigener Lebensmeister sein, und mehr als mich selbst ist nicht zu haben. Ich muss mein eigener Kraftspender, Lehrer und Tröster sein. Ich muss der Bäcker meiner eigenen Lebensbrote sein.» ${ }^{16}$ Das aber wäre eine zwar unabhängige, autarke, aber trostlose Existenz. Demgegenüber hält es Steffensky geradezu für schön und lebenserleichternd, angewiesen zu sein und sich dafür im Modus des Empfangens wahrnehmen zu können, sich vielleicht sogar ein Stück weit aus der Hand geben und der Sorge anderer anvertrauen zu dürfen. Darin liegt eine mögliche Quelle von Sinnerfahrung gerade in der Erfahrung eigener Abhängigkeit. Allerdings fügt Steffensky dieser Aussage die Bemerkung hinzu: «Es ist eine schwere Kunst, bedürftig zu sein und sich trösten zu lassen, die Kunst der Passivität. Viel Erwachsenheit gehört zu ihr, vielleicht auch viele Lebensniederlagen.» Und darum dürfte man im Alter eher Zugang zu dieser Kunst finden als in jüngeren Jahren.

Die Kunst der Abhängigkeit oder der Passivität fällt uns nicht leicht; sie wird in modernen westlichen Gesellschaften nicht kultiviert. Das kulturelle Wertesystem erklärt Passivität und Abhängigkeit vielmehr zu etwas, das vermieden oder überwunden werden muss. Häufig sind es wohl in der

16 Fulbert Steffensky, Der alltägliche Charme des Glaubens, Würzburg 2002, 81.
Jahrbuch Diakonie Schweiz 4 (2020/2021) http://dx.doi.org/10.22018/JDS.2021.16 
Tat persönliche Erfahrungen von Grenzen, von Krankheit oder von altersbedingten Problemen, die uns allererst zu einer «erwachsenen», reifen Auseinandersetzung mit einer Kultur der Abhängigkeit oder der Passivität nötigen.

\section{Abhängigkeit als Phänomen des hohen Alters}

Abhängigkeit ist ein Grundphänomen allen Lebens. Es manifestiert sich allerdings besonders deutlich im Kindesalter und in der Phase der Hochaltrigkeit. Während bei heranwachsenden Kindern Abhängigkeit als vorübergehendes Phänomen als normal gilt, nimmt sie bei Hochbetagten zu und wird von der Gesellschaft und den Betroffenen selbst als gravierendes Problem wahrgenommen. In einem Artikel, in dem sie Einsichten aus rund 20 Jahren ihrer psychogerontologischen Forschung zusammenfasste, bemerkte Margret M. Baltes schon vor Jahren, dass Abhängigkeit eines der bedrückendsten Probleme im Alter darstelle. ${ }^{17}$ Und die Gerontologin Martha B. Holstein und Kollegen haben darauf hingewiesen, dass Abhängigkeit im hohen Alter oft als Ausdruck eines Würdeverlustes und eines mangelnden Selbstwertes empfunden wird - mit entsprechend negativen Auswirkungen auf alte Menschen. ${ }^{18}$ Je mehr diese bei fortschreitender Gebrechlichkeit auf Hilfen in den Aktivitäten des täglichen Lebens (kochen, putzen, ankleiden, Toilettengang etc.) angewiesen sind, desto peinlicher

17 Margret. M. Baltes, The many faces of dependency in old age, Cambridge 1996, 1.

18 Holstein / Parks / Waymack, Ethics, aging, and society (Anm. 13), 12: «Dependence has been strongly associated with weakness, incapacity, neediness and a lack of dignity; insofar as individuals are able to resist dependence, they are able to maintain their dignity and self-respect. But this strong emphasis on autonomy as independence has had a very negative impact on aging and aged persons, who find themselves increasingly in need of assistance to bathe, to go to the bathroom, dress, eat, and get about. It is seen to be shameful and embarrassing to admit that you can no longer perform all these tasks unassisted.» und beschämender wird Abhängigkeit von ihnen erlebt. Sie erfahren sich als minderwertig gemessen an den zentralen Werten des mainstreams der Gesellschaft wie etwa Autonomie, Produktivität und Aktivität.

Gerade angesichts der zunehmenden Zahl hochaltriger Menschen ist es darum gesellschaftlich von zentraler Bedeutung, ein Verständnis von Menschsein zu entwickeln, bei dem Abhängigsein von anderen kein Makel ist, sondern genau so zentral zu gelingendem, würdevollem Leben gehört wie Unabhängigkeit. Es muss wieder deutlich werden, dass eine menschenfreundliche Lebenskunst nur unter Einschluss einer «Kunst der Abhängigkeit» (Riedel) bzw. einer «Kunst der Passivität» (Steffensky) zu gewinnen ist. In diesem Sinne plädiert der deutsche Gerontologe Andreas Kruse seit Jahren eindringlich dafür, Selbstständigkeit, Selbstverantwortung, Mitverantwortung und bewusst angenommene Abhängigkeit gleichwertig als zentrale ethische Leitkategorien eines gelingenden Lebens im Älterwerden ernst zu nehmen. ${ }^{19}$

\section{Autor:}

Dr. Heinz Rüegger, freischaffender Theologe, Ethiker und Gerontologe und freier Mitarbeiter im Institut Neumünster
Jahrbuch Diakonie Schweiz 4 (2020/2021) - ISSN 2504-3994 CC by 4.0
Jahrbuch Diakonie Schweiz 4 (2020/2021) http://dx.doi.org/10.22018/JDS.2021.16 